\title{
Assessment of Flex-Grid/SDM Backbone Networks under Inter-Core XT-limited Transmission Reach
}

\author{
Jordi Perelló, Joan M. Gené, Jose A. Lazaro, Albert Pagès, and Salvatore Spadaro \\ Universitat Politècnica de Catalunya (UPC) - Barcelona Tech \\ Jordi Girona 1-3, 08034 Barcelona, Spain \\ e-mail: perello@ac.upc.edu, \{jgene,jose.lazaro, albertpages, spadaro\}@tsc.upc.edu
}

\begin{abstract}
In the present paper, we estimate the transmission reach of the optical signals through several state-of-the-art multicore fibers reported in the literature considering ASE noise and inter-core crosstalk. Using these estimations, we next assess the resource efficiency of Flex-grid/SDM backbone network deployments using the considered multi-core fibers against currently available multi-fiber link solutions.
\end{abstract}

Keywords-Flex-grid, SDM, multi-core fibers, crosstalk.

\section{INTRODUCTION}

Spatial division multiplexing (SDM) is the only foreseen solution to overcome the nonlinear Shannon limit of standard single-mode fibers [1]. Multi-core fiber (MCF) technology is a promising candidate for SDM due to the extremely low intercore crosstalk (XT) in up to 19 cores [2][3][4]. To the best of our knowledge, detailed offline planning studies for MCFenabled backbone transport networks including modulation format-specific XT limitations are unavailable in the literature. Following the common practice of network operators to plan their networks based on worst-case Transmission Reach (TR), this paper derives novel TR estimations over 7, 12 and 19-core MCFs, accounting for optical signals at 40, 100 and $400 \mathrm{~Gb} / \mathrm{s}$ employing multiple modulation formats with different spectral efficiencies (from BPSK to 64-QAM, assuming polarization multiplexing). We should note that our XT extrapolations are not based on theoretical values like the ones used for example in [5], but on real measurements from available state-of-the-art MCF prototypes with 7,12 and 19 cores (reported in references [2], [3] and [4], respectively).

To maximize the spectrum utilization in each of the MCF cores, we assume Flex-Grid technology instead of conventional DWDM, given its enhanced flexibility in allocating both subwavelength connections and high capacity super-channels over the cores' available spectral resources. As we will show later on through simulation results, Flex-Grid/SDM backbone networks deploying MCFs with moderate number of cores (7, 12) can provide very similar resource utilization as with multifiber links, while opening the possibility to cost-effective integrated system components for MCFs, like those envisioned in [6]. In scenarios with MCFs with high number of cores (19), reasonable resource utilization degradation from $6-19 \%$ is still observed against multi-fiber link solutions, depending of the traffic profile and physical link distances.

\section{INTER-CORE CROSSTALK}

The TR of a single-core optically-amplified link can be limited by different factors. Considering that chromatic dispersion and polarization-mode dispersion are compensated by usual technical solutions (chromatic dispersion compensating fibers or appropriate compensating algorithms in coherent receivers), the remaining relevant impairments are amplified spontaneous emission (ASE) noise and fiber nonlinearities. To avoid reaching the nonlinear regime it is common to fix the maximum optical power per channel below $1 \mathrm{~mW}$. The required Signal-to-Noise-Ration (SNR) at the receiver is then determined by the modulation format, finally establishing the available power budget. The maximum transmission distance limited by ASE noise is:

$$
L_{\text {max }, S N R}=\frac{P_{s} \cdot L_{\text {span }}}{S N R_{\min } \cdot h \cdot f \cdot G \cdot N F \cdot R_{s}},
$$

where $P_{s}$ is the optical power at the transmitter, $L_{\text {span }}$ is the distance between amplifiers, $S N R_{\min }$ is the required SNR at the receiver side (as in the examples in Tab. I), $G$ the gain of the amplifiers, $N F$ the noise factor of the amplifiers, and $R_{S}$ the symbol rate (optimum Nyquist pulses are assumed [1]).

TABLE I. THEORETICAL REQUIRED SNR AT BER OF $10^{-3}$ [1]

\begin{tabular}{cccc}
\hline \hline BPSK & QPSK & 16-QAM & 64-QAM \\
\hline $6.7 \mathrm{~dB}$ & $9.7 \mathrm{~dB}$ & $16.5 \mathrm{~dB}$ & $22.5 \mathrm{~dB}$ \\
\hline
\end{tabular}

Moreover, transmission through MCFs is affected by intercore crosstalk (XT), which may become the limiting factor. Some worst aggregate inter-core XT values (measured at 1550 $\mathrm{nm}$ and referenced to $1 \mathrm{~km}$ of fiber) are shown in Tab. II for state-of-the-art MCFs in the literature.

TABLE II. WORST AGGREGATE INTER-CORE XT

\begin{tabular}{ccc}
\hline \hline 7 cores [2] & 12 cores $[3]$ & 19 cores $[4]$ \\
\hline$-84.6 \mathrm{~dB}$ & $-61.9 \mathrm{~dB}$ & $-54.6 \mathrm{~dB}$ \\
\hline
\end{tabular}

The maximum transmission distance limited by inter-core crosstalk reads [2][3][4]:

$$
L_{\text {maax }, X T}=10^{\frac{X T_{d B, \min }-X T_{d B, 1 \mathrm{kn}}}{10}}[\mathrm{~km}],
$$


where $X T_{d B, \min }$ and $X T_{d B, 1 \mathrm{~km}}$ refer to the required minimum crosstalk value and to the fibre's inter-core XT referenced to $1 \mathrm{~km}$, respectively. Both quantities are given in $\mathrm{dB} . X T_{d B \text {, min }}$ depends on the modulation format used (see Tab. III).

TABLE III. IN-BAND XT VALUES AT 1DB-PENALTY [7]

\begin{tabular}{cccc}
\hline \hline BPSK & QPSK & 16-QAM & 64-QAM \\
\hline$-14 \mathrm{~dB}$ & $-17 \mathrm{~dB}$ & $-23 \mathrm{~dB}$ & $-29 \mathrm{~dB}$ \\
\hline
\end{tabular}

TR estimations are summarized in Tab. IV. Calculation parameters are provided below the table. A $4 \mathrm{~dB}$ margin is included in both the SNR and XT limit values. Polarization multiplexing (PM) and a 12\%-overhead forward-error correction (FEC) are assumed. White and grey cells in the table show noise-limited and XT-limited cases, respectively. The limitation induced by noise is inversely dependant to the bit rate while the impact of crosstalk is independent. As shown, the higher the bit rate, the more limited the reach by noise.

TABLE IV. TRANSMISSION REACH VALUES (IN KM)

\begin{tabular}{|c|c|c|c|c|c|}
\hline $\begin{array}{c}\text { Bit } \\
\text { Rate }\end{array}$ & $\begin{array}{l}\text { No. of } \\
\text { Cores }\end{array}$ & BPSK & QPSK & 16-QAM & 64-QAM \\
\hline \multirow{3}{*}{$\begin{array}{c}40 \\
\text { Gb/s }\end{array}$} & 7 & 8345 & 8365 & 3495 & 1317 \\
\hline & 12 & 8345 & 8365 & 3062 & 769 \\
\hline & 19 & 4755 & 2383 & 599 & 150 \\
\hline \multirow{3}{*}{$\begin{array}{c}100 \\
\text { Gb/s }\end{array}$} & 7 & 3338 & 3346 & 1398 & 527 \\
\hline & 12 & 3338 & 3346 & 1398 & 527 \\
\hline & 19 & 3338 & 2383 & 599 & 150 \\
\hline \multirow{3}{*}{$\begin{array}{c}400 \\
\text { Gb/s }\end{array}$} & 7 & 835 & 837 & 350 & 132 \\
\hline & 12 & 835 & 837 & 350 & 132 \\
\hline & 19 & 835 & 837 & 350 & 132 \\
\hline
\end{tabular}

$$
\begin{array}{ll}
P_{s}=1 \mathrm{~mW} & \mathrm{G}=20 \mathrm{~dB} \\
L_{\text {span }}=100 \mathrm{~km} & \mathrm{NF}=5.5 \mathrm{~dB} \\
\lambda=1550 \mathrm{~nm} &
\end{array}
$$

\section{FLEX-GRID/SDM OFFLINE NETWORK PLANNING}

Heuristic approaches seem to be the only computationally tractable solution to achieve the planning of Flex-Grid/SDM transport networks, given the large amount of traffic demands that they are able to carry. In this work, we propose a heuristic for the offline Routing, Modulation, Core and Spectrum Assignment (RMCSA) problem in such kind of networks. That is, we aim at assigning the best route, modulation format, core and spectrum portion to each of the offered demands, so that the highest Frequency Slot (FS) allocated in any core of any MCF in the network is minimized. Our heuristic, called Cumulative RMCSA (C-RMCSA) extends the SPSA heuristic proposed in [5] for the simpler offline Routing Core and Spectrum Assignment (RCSA) problem, to also decide on the most appropriate modulation format for each demand.

Specifically, C-RMCSA serves all the offered demands (no blocking) iteratively in a greedy fashion as follows:

1. For each offered demand, compute its set of candidate paths (in this work, the 3 shortest paths in $\mathrm{km}$ between its source-destination nodes). For each candidate path, find the most efficient feasible modulation format at the demand bit rate, given the TR estimations shown before in Tab. IV and the length of the path in $\mathrm{km}$.

2. Sort the list of offered demands according to their minimum required number of FS (the number of FS required through their shortest candidate path) in descending order (demands requiring more FSs are sorted first).

3. While the list of offered demands pending to be served is not empty:

1. Set $\max F S$ (maximum reservable FS in any core of any MCF) to its value in the previous iteration (initially 0 ) plus the number of FS needed to allocate the first pending demand in the list (the most demanding one in terms of FS).

2. Iterate the list of offered demands pending to be served from the first to the last one, trying to allocate them on any of their candidate paths using the chosen modulation format (for the core and spectrum assignment, a First-Fit strategy is used, ensuring both slot contiguity and continuity constraints along the path. Note that core continuity is unnecessary as intermediate nodes can switch signals among i/o cores). If a demand can be served, reserve the required FS along the selected candidate path and remove this demand from the list.

Following this iterative process, C-RMCSA succeeds in balancing the established connections over the network, thus achieving good spectrum utilization, while keeping the highest FS used in the network low, that is, the main objective in our Flex-Grid/SDM network planning.

\section{RESULTS AND DISCUSSION}

In this section we use the proposed C-RMCSA heuristic to perform the planning of a National and a European-wide FlexGrid/SDM transparent network. Then, we compare the required number of FS against a benchmark Multi-Fibre link (MF) network solution, where inter-core XT does not apply (i.e., TR in MF scenarios is assumed to only be limited by ASE noise, e.g., $8345 \mathrm{~km}$ for $40 \mathrm{~Gb} / \mathrm{s}$ BPSK in all 7, 12 and 19 MF scenarios, $8365 \mathrm{~km}$ for $40 \mathrm{~Gb} / \mathrm{s}$ QPSK, etc.). Specifically, we consider the 12-Node with 20 links Deutsche Telekom (DT) network and an 11-Node with 18 links PanEuropean Network (EON), whose topologies and physical link distances are found in [8]. Average link distances are $243 \mathrm{~km}$ and $526 \mathrm{~km}$ in the DT and EON, respectively, hence permitting our Flex-Grid/SDM to be checked under different backbone network conditions and thus inter-core XT effects. As for the available spectrum, we assume the entire C-Band 4 $\mathrm{THz}$, discretized in $12.5 \mathrm{GHz} \mathrm{FS}$, leading to $320 \mathrm{FS}$ available per core/SCF in the MCFs/MF scenarios. Moreover, we assume $10 \mathrm{GHz}$ guard-bands between adjacent connections. 
We consider 2 offered traffic profiles, TP-1 and TP-2, aiming to simulate short-term and mid-term network scenarios. In TP-1, offered demands are of 40, 100 and 400 $\mathrm{Gb} / \mathrm{s}$, with probabilities of $0.3,0.5$ and 0.2 , respectively. In TP-2 they are of 100 and $400 \mathrm{~Gb} / \mathrm{s}$, with respective probabilities of 0.4 and 0.6 .

The quite low TR values of the modulation formats at 400 $\mathrm{Gb} / \mathrm{s}$ (Tab. IV) can prevent demands to reach their destination transparently. Only when a $400 \mathrm{~Gb} / \mathrm{s}$ demand is unfeasible due to TR even with the least efficient modulation format, we serve it as $4 \times 100 \mathrm{~Gb} / \mathrm{s}$ connections, contiguously allocated and jointly switched from source to destination. Thus, the total required number of FS is 4 times those of the employed modulation format at $100 \mathrm{~Gb} / \mathrm{s}$ (i.e., including therefore 4 guard-bands). Fig. 1 shows in the DT (top) and EON (bottom) networks the highest allocated FS in any core/fibre of the $\mathrm{MCF} / \mathrm{MF}$ network scenario with 7, 12 and 19 cores/fibres per link, Specifically, 3k, 5k and $8 \mathrm{k}$ uniformly distributed demands are offered to the MCF/MF scenarios with 7, 12, 19 cores/fibres, respectively (following either TP-1 or TP-2). Each result is obtained averaging 20 executions, generating a random demand set each time. For better comparison, the same set is offered to the MCF and respective MF scenario in each execution.
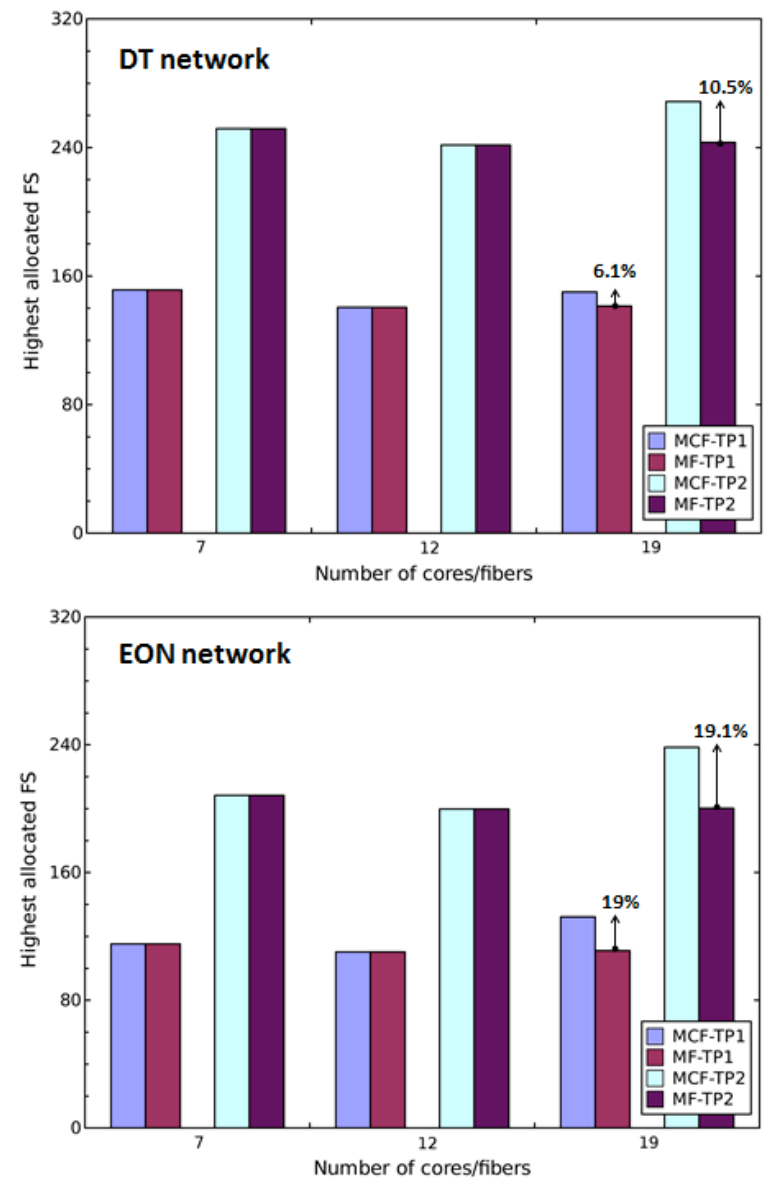

Fig. 1. Highest allocated FS the DT (top) and EON (bottom) networks, with 7, 12 and 19 cores/fibers per link. Differences beween MCF and MF scenarios are observed only with 19 cores/fibers per link.
As depicted, no required FS differences exist between 7 and 12 core/fiber MFC/MF scenarios. This is because the TR in the considered 7 and 12-core MCFs is mostly limited by ASE noise (see Tab. IV). The only cases where TR is intercore XT-limited are $40 \mathrm{~Gb} / \mathrm{s}$ 16-QAM and 64-QAM. However, with the assumed (typical) values of FS width and guard-bands, both options equally require 2 FSs. Hence, even if $40 \mathrm{~Gb} / \mathrm{s}$ 64-QAM is feasible for a demand in the MF scenario, and it has to be lowered to 16-QAM with MCFs for TR issues, the same number of FSs are used. Looking at the $\mathrm{MCF} / \mathrm{MF}$ scenarios with 19 cores/fibres, more significant differences are observed, particularly in the EON, with larger physical link distances. Major TR limitations due to inter-core $\mathrm{XT}$ are observed at $100 \mathrm{~Gb} / \mathrm{s}$ bit-rates, which may force 100 $\mathrm{Gb} / \mathrm{s}$ connections using 16-QAM in the MF scenario to be lowered to QPSK with MCFs, requiring more FSs. Furthermore, even that modulation formats at $400 \mathrm{~Gb} / \mathrm{s}$ are all limited by ASE noise, some demands eventually have to be supported over $4 \times 100 \mathrm{~Gb} / \mathrm{s}$ connections, affected by inter-core XT too. For these reasons, around $6-10 \%$ and $19 \%$ increments of required FS in the DT and EON with 19-core MCFs are observed against the respective MF benchmark.

\section{CONCLUSIONS}

The obtained results suggest that inter-core XT is not an issue in MCF-enabled optical backbone networks when the number of cores is moderate (e.g., 7 or 12). Even with 19-core MCFs, an assumable 19\% increase of FS is observed against the equivalent MF scenario. This outcome motivates FlexGrid/SDM even in large backbone networks, which would benefit from cost-effective integrated system components as transponders, amplifiers, ROADMs, etc., as envisioned in [6].

\section{ACKNOWLEDGEMENT}

This work has been partially funded by the Spanish National Project SUNSET (TEC2014-59583-C2-1-R).

\section{REFERENCES}

[1]. R.-J. Essiambre et al., "Capacity Limits of Optical Fiber Networks", J. Lightwave Technol., Vol. 28, no. 4, pp. 662-701, Feb. 2010.

[2]. J. Sakaguchi et al., "Space Division Multiplexed Transmission of 109$\mathrm{Tb} / \mathrm{s}$ Data Signals Using Homogeneous Seven-Core Fiber", J. Lightwave Technol., Vol. 30, no. 4, p. 658-665, Jan. 2012.

[3]. Sano et al., "409- $\mathrm{Tb} / \mathrm{s}+409-\mathrm{Tb} / \mathrm{s}$ crosstalk suppressed bidirectional MCF transmission over $450 \mathrm{~km}$ using propagation-direction interleaving," Opt. Express, Vol. 21, pp. 16777-16683, Jul. 2013.

[4]. J. Sakaguchi et al., "19-core MCF transmission system using EDFA with shared core pumping coupled via free-space optics," Opt. Express, Vol. 22, pp. 90-95, Jan. 2014.

[5]. Muhammad et al., "Routing, Spectrum and Core Allocation in Flexgrid SDM Networks with Multi-core Fibers", Proc. ONDM 2014, Stockholm (Sweden), May 2014.

[6]. P. J. Winzer, "Spatial Multiplexing in Fiber Optics: The 10x Scaling of Metro/Core Capacities," Bell Labs Tech. Journal, Vol.19, pp. 22-30, Sept. 2014.

[7]. P. J. Winzer, "Spatial Multiplexing: The Next Frontier in Network Capacity Scaling," in Proc. European Conference Exhibition Optical Communication, Paper We.1.D.1, London, U.K., 2013.

[8]. J. Perelló et al., "Power consumption reduction through elastic data rate adaptation in survivable multi-layer optical networks", Photon. Netw. Commun., Vol. 28, pp. 276-286, Dec. 2014. 